\title{
Optimizing Prescription of Chinese Herbal Medicine for Unstable Angina Based on Partially Observable Markov Decision Process
}

\author{
Yan Feng, ${ }^{1}$ Yu Qiu, ${ }^{2}$ Xuezhong Zhou, ${ }^{3}$ Yixin Wang, ${ }^{1}$ Hao Xu, ${ }^{2}$ and Baoyan Liu ${ }^{4}$ \\ ${ }^{1}$ Department of General Practice, Anzhen Hospital, Capital Medical University, Beijing 100029, China \\ ${ }^{2}$ Department of Cardiology, Xiyuan Hospital, China Academy of Chinese Medical Sciences, Beijing 100091, China \\ ${ }^{3}$ School of Computer and Information Technology, Beijing Jiaotong University, Beijing 100044, China \\ ${ }^{4}$ China Academy of Chinese Medical Sciences, Beijing 100700, China \\ Correspondence should be addressed to Hao Xu; xuhaotcm@hotmail.com and Baoyan Liu; liuby@mail.cintcm.ac.cn
}

Received 5 May 2013; Accepted 4 July 2013

Academic Editor: Keji Chen

Copyright (C) 2013 Yan Feng et al. This is an open access article distributed under the Creative Commons Attribution License, which permits unrestricted use, distribution, and reproduction in any medium, provided the original work is properly cited.

\begin{abstract}
Objective. Initial optimized prescription of Chinese herb medicine for unstable angina (UA). Methods. Based on partially observable Markov decision process model (POMDP), we choose hospitalized patients of 3 syndrome elements, such as qi deficiency, blood stasis, and turbid phlegm for the data mining, analysis, and objective evaluation of the diagnosis and treatment of UA at a deep level in order to optimize the prescription of Chinese herb medicine for UA. Results. The recommended treatment options of UA for $q i$ deficiency, blood stasis, and phlegm syndrome patients were as follows: Milkvetch Root + Tangshen + Indian Bread + Largehead Atractylodes Rhizome (ADR = 0.96630); Danshen Root + Chinese Angelica + Safflower + Red Peony Root + Szechwan Lovage Rhizome Orange Fruit $(A D R=0.76)$; Snakegourd Fruit + Longstamen Onion Bulb + Pinellia Tuber + Dried Tangerine peel + Largehead Atractylodes Rhizome + Platycodon Root $(A D R=0.658568)$. Conclusion. This study initially optimized prescriptions for UA based on POMDP, which can be used as a reference for further development of UA prescription in Chinese herb medicine.
\end{abstract}

\section{Introduction}

Unstable angina (UA) is the clinical status between exertional stable angina and acute myocardial infarction [1]. In recent years, traditional Chinese medicine (TCM) got wealthy experience and remarkable achievements in the treatment of UA. However, there are still problems in how to scientifically evaluate the clinical efficacy of different clinical treatment recommendations and how to convert many personalized experiences into certain standardized treatment plans to follow.

Treatment regimen optimization based on partially observable Markov decision process (POMDP) is the course of using scientific methods of computation to find the most economical and convenient treatment program with best clinical efficacy among many treatment options [2-4]. The certain amount of knowledge accumulated and discovered from the massive clinical data and TCM treatment experience can not only verify the existing experience and theory of
TCM that may also find some new treatment experience. In this study, we will try to use data mining methods based on large-scale, nonexternal control observational clinical data in practice to seek and find the optimized TCM treatment prescription.

\section{Materials and Methods}

2.1. Subjects. From September 2009 to February 2011, a total of 2212 hospitalized subjects were enrolled from China-Japan Friendship Hospital affiliated to National Health and Family Planning Commission (Beijing, China) (589 cases), Xiyuan Hospital affiliated to China Academy of Chinese Medical Science (Beijing, China) (362 cases), Guang Anmen Hospital affiliated to China Academy of Chinese Medical Science (Beijing, China) (298 cases), Wangjing Hospital affiliated to China Academy of Chinese Medical Science (Beijing, China) (97 cases), Dongzhimen Hospital affiliated to Beijing 
University of Chinese Medicine (Beijing, China) (193 cases), Beijing Integrative Medicine Hospital (Beijing, China) (121 cases), Beijing Chinese Medicine Hospital affiliated to Capital Medical University (Beijing, China) (186 cases), Beijing Anzhen Hospital affiliated to Capital Medical University (Beijing, China) (42 cases), Beijing Tongren Hospital affiliated to Capital Medical University (Beijing, China) (43 cases), People's Hospital affiliated to Peking University (Beijing, China) (41 cases), Huairou District Hospital of Traditional Chinese Medicine (Beijing, China) (138 cases), and Tongzhou District Hospital of Traditional Chinese Medicine (Beijing, China) (102 cases). All selected subjects fulfilled the diagnosis of unstable angina. Ethical approval was granted by the Ethics Committee of China-Japan Friendship Hospital (Beijing, China). Informed consent was obtained by each patient participating in this study.

2.2. Diagnostic Standard. UA diagnostic standard referred to the UA diagnosis and treatment recommendations by the China Cardiovascular Association released in 2007 [5]. According to the characteristics of angina pectoris, typical ECG changes, exercise treadmill ECG, Holter, cardiac scintigraphy, coronary angiography and risk elements to make the judgment in order to improve the accuracy of diagnosis. Diagnostic standards of TCM syndromes referred to the following references: (1) the TCM standards of coronary heart disease by China Association of Integrative Medicine Association for cardiovascular diseases [6] and (2) the chest stuffiness and pains dialectical standards in Chinese internal medicine [7].

2.3. Inclusion Criteria. The inclusion criteria were as follows: (1) meet the diagnostic criteria; (2) previous history of old myocardial infarction or coronary angiography confirmed at least one coronary stenosis $\geq 50 \%$, (3) hospitalized patients with UA as first western medical diagnosis, (4) age, sex, druged use, and concomitant diseases are not limited, and (5) a signed informed consent.

2.4. Exclusion Criteria. The exclusion criteria were composed of five conditions: (1) occurred end-point events in 1-year fellow, (2) cancer and immune system diseases, (3) pregnant or lactating women, (4) serious diseases of liver, kidney, and hematopoietic system, and (5) patients with allergies or psychosis.

2.5. End-Point Events Criteria. (1) The primary end-point events are cardiovascular death, nonfatal myocardial infarction, revascularization (including intervention, coronary artery bypass grafting); (2) secondary end-point events are stroke, rehospitalization for ACS, heart failure, and other thrombotic complications.

2.6. Western Medical Treatment. According to the UA diagnosis and treatment recommendations by China Cardiovascular Association released in 2007 [5], patients were given conventional western drug therapy including antiischemic (nitrates, beta-blockers, calcium antagonists, and
ACE inhibitors), antiplatelet (aspirin and/or clopidogrel), anticoagulation (heparin or low molecular weight heparin), and statin drugs.

2.7. Observed Indicators and Methods. Doing clinical information collection, verification, supplementary, pre-processing and data mining, and analysis for data acquisition process in accordance with the unified design of UA. Using TCM clinical research data platform for individualized treatment, qualified clinical researchers after training and examination took patients' hospital stay information and entered them into the database through clinical information collection system; then the professionals of the school of Computer Science in Beijing Jiaotong University convert the data for extraction, washing, and analysis. To specify the TCM syndrome following the rules of "Diagnostics of Chinese Medicine" [8] and "TCM syndrome differential diagnosis" [9], such as "phlegm stagnation syndrome," "phlegm dampness syndrome," "phlegm stasis syndrome" unified as "turbid phlegm syndrome," break down the combined TCM syndromes into basic TCM syndrome elements. For example, break down "qi and yin deficiency syndrome" into syndrome elements as "qi deficiency syndrome" and "yin deficiency syndrome." When encountering difficulty in distinguishing and differentiating TCM syndromes, to discuss and resolve it under the guidance of experts and references, telephone followups were enrolled one year after the end of the event.

In order to simplify the data and find common rules, we elected the core prescription medicine as the object of analysis, and the extraction of core Chinese herbal medicine applied the complex network mining method. To divide five main symptoms of patients with UA: "chest tightness, chest pain, heart palpitations, shortness of breath, and fatigue" into "no, light, medium, and heavy" four grades and to observe the changes of the main symptoms for patients during hospitalization every 1-2 days after admission time with 5 times for each, use cluster analysis method to unify symptoms.

2.8. Statistical Analysis. Using Oracle $9.0 \mathrm{~g}$ to converse the demographic data, clinical features, syndromes, and drug treatment data, while general material use frequency statistical analysis. Getting use of the data mining platform built in the major project of the Beijing Municipal Science and Technology Commission for the prevention and treatment of major diseases to optimize the prescription of Chinese herbal medicine and doing POMDP mining analysis by Beijing Jiaotong University researchers with data mining tools. The level of average discounted reward (ADR) will take the degree of main symptoms improvement as a standard to evaluate the clinical efficacy of different treatment options.

\section{Results}

3.1. The Demographic Information of the Subjects. A total of 2212 (1341 males and 871 females) cases of subjects, aged more than 40 years, were included in this study. This group of subjects included 1061 cases of patients complicated with 
TABLE 1: The demographic information of the subjects.

\begin{tabular}{lc}
\hline Items & Demographic information \\
\hline Gender & \\
$\quad$ Males (case (\%)) & $1341(60.6 \%)$ \\
Females (case (\%)) & $871(39.4 \%)$ \\
Age (years) & $61.2 \pm 13.6$ \\
Hypertension (case (\%)) & $1061(48.0 \%)$ \\
Diabetes & $514(23.2 \%)$ \\
Hyperlipemia & $513(23.1 \%)$ \\
Old myocardial infarction & $328(14.8 \%)$ \\
Cerebral infarctions & $219(9.9 \%)$ \\
\hline
\end{tabular}

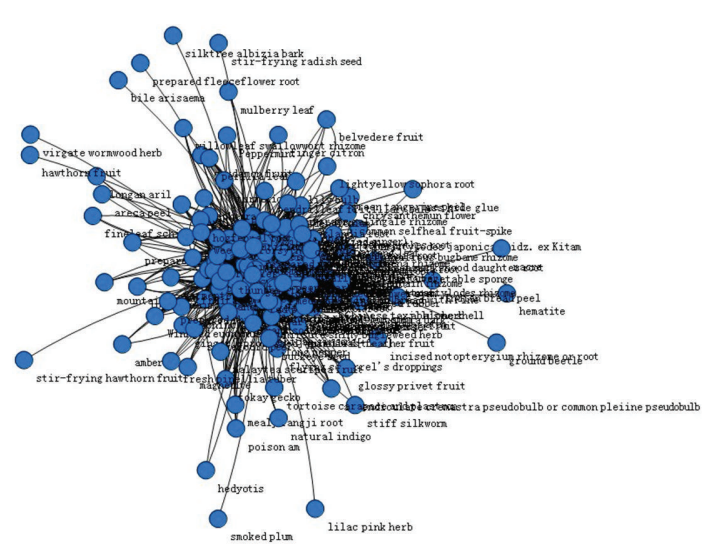

FIGURE 1: Overall situation of Chinese herbal prescription medicine for TCM syndrome element " $q i$ Deficiency."

hypertension, 514 cases of patients complicated with diabetes, 513 cases of patients complicated with hyperlipemia, 328 cases of patients complicated with old myocardial infarction, and 219 cases of patients complicated with cerebral infarctions. The demographic data is shown in Table 1.

3.2. TCM Syndrome Distribution in Patients with UA. Among the 2212 UA patients, the common Chinese syndrome elements were as follows: blood stasis (1931 cases, 87.3\%), qi deficiency (1140 cases, 51.5\%), turbid phlegm (1059 cases, $47.9 \%$ ), yin deficiency (412 cases, $18.6 \%$ ), qi stagnation (148 cases, $6.7 \%$ ), yang deficiency (65 cases, $2.9 \%$ ), heat (60 cases, $2.7 \%$ ), and blood deficiency (23 cases, $1.0 \%$ ).

3.3. Core Medicine of Chinese Herbal Prescription (Figures 1, 2, 3, and Table 2). Due to the requirements in the number of patients for model, we study the treatment options for patients with UA in three TCM syndrome elements as "qi deficiency," "blood stasis," and "turbid phlegm." Use complex network clustering method to screen the core Chinese herbal medicine of three TCM syndrome elements. With all the Chinese herbal medicine used in the treatment options of a syndrome element as the nodes, the medicine in compatibility has "interconnected" feature, and the number of "interconnected" is the medicine "related frequency". The most frequently related nodes with other medicine have the most critical role in all the medication of this syndrome

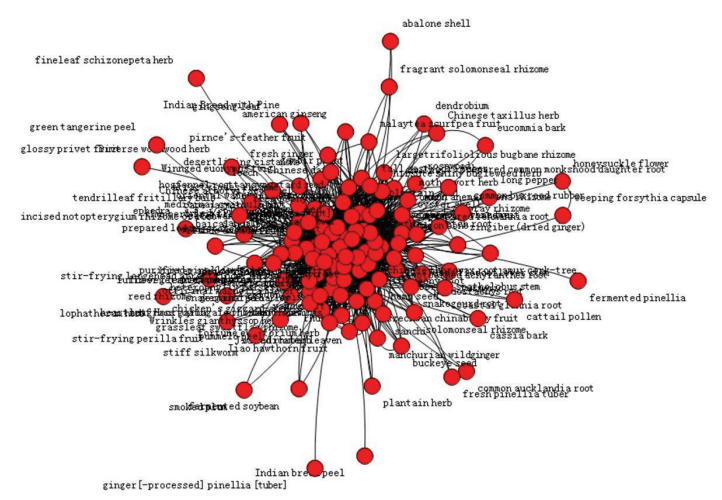

FIGURE 2: Overall situation of Chinese herbal prescription medicine for TCM syndrome element "blood stasis."

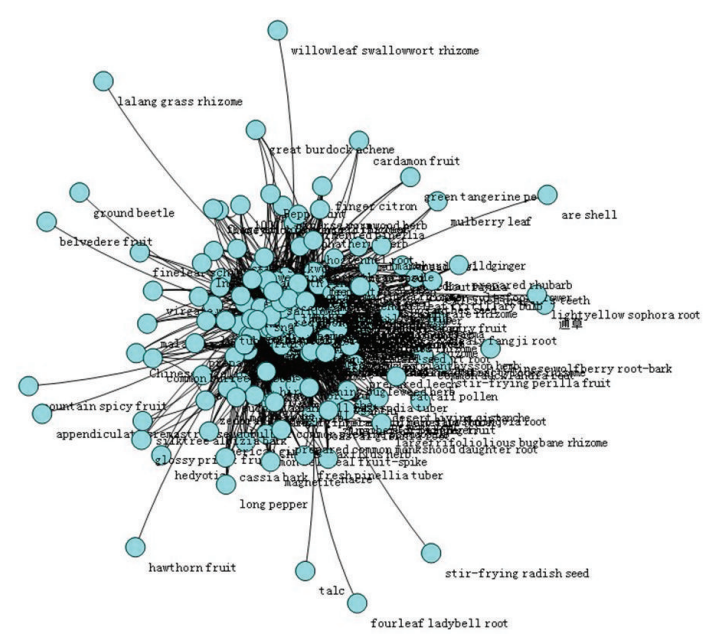

FIGURE 3: Overall situation of Chinese herbal prescription medicine for TCM syndrome element "turbid phlegm."

element. Thus, the densest nodes are the core prescription medicine.

3.4. Optimization and Efficacy Evaluation of Prescriptions for Different TCM Syndrome Elements in Patients with UA (Table 3). Applying POMDP model to calculate the ADR of different prescriptions, the largest ADR prescription of TCM syndrome element can get the maximum benefit in symptom improvement and long-term efficacy. Among the prescriptions for 3 different TCM syndrome elements, the combination of "Milkvetch Root + Tangshen + Indian Bread + Largehead Atractylodes Rhizome" is the optimizing prescription of TCM syndrome element "qi deficiency" for patients with UA, the combination of "Danshen Root + Chinese Angelica + Safflower + Red Peony Root + Szechwan Lovage Rhizome, Orange Fruit" is the optimizing prescription of TCM syndrome element "blood stasis" for patients with UA, and the combination of "Snakegourd Fruit + Longstamen Onion Bulb + Pinellia Tuber + Dried Tangerine peel + Largehead Atractylodes Rhizome + Platycodon Root" 
TABLE 2: Core medicine of Chinese herbal prescription.

\begin{tabular}{|c|c|}
\hline $\begin{array}{l}\text { Types of TCM } \\
\text { syndrome element }\end{array}$ & Core medicine of Chinese herbal prescription \\
\hline Qi deficiency & $\begin{array}{l}\text { Tangshen, Heterophylly Falsestarwort Root, Largehead Atractylodes Rhizome, Milkvetch Root, Radix } \\
\text { Glycyrrhizae, Indian Bread, and Chinese Date }\end{array}$ \\
\hline Turbid phlegm & $\begin{array}{l}\text { Pinellia Tuber, Snakegourd Fruit, Indian Bread, Dried Tangerine peel, Longstamen Onion Bulb, Largehead } \\
\text { Atractylodes Rhizome, Platycodon Root, Golden Thread, Bamboo Shavings, Wrinkled Giant Hyssop + Herba } \\
\text { Eupatorii, Wrinkled Giant Hyssop, Orange Fruit + Pinellia Tuber }\end{array}$ \\
\hline Blood stasis & $\begin{array}{l}\text { Szechwan Lovage Rhizome, Yanhusuo, Danshen Root, Peach Seed, Safflower, Chinese Angelica, Turmeric Root } \\
\text { Tuber, Red Peony Root, Turmeric Root Tuber + Red Peony Root, Peach Seed + Radix bupleuri, Peach Seed + } \\
\text { Platycodon Root, Szechwan Lovage Rhizome + Orange Fruit }\end{array}$ \\
\hline
\end{tabular}

TABLE 3: Optimizing prescription of different TCM syndrome elements for UA patients.

\begin{tabular}{lcc}
\hline $\begin{array}{l}\text { Types of TCM } \\
\text { syndrome slement }\end{array}$ & Optimizing prescription & ADR \\
\hline Qi deficiency & Milkvetch Root + Tangshen + Indian Bread + Largehead Atractylodes Rhizome & 0.96630 \\
\hline Blood stasis & Danshen Root + Chinese Angelica + Safflower + Red Peony Root + Szechwan Lovage Rhizome Orange & 0.76 \\
\hline $\begin{array}{l}\text { Furbid phlegm } \\
\text { Fult }\end{array}$ & Snakegourd Fruit + Longstamen Onion Bulb + Pinellia Tuber + Dried Tangerine peel + Largehead \\
Atractylodes Rhizome + Platycodon Root & 0.658568 \\
\hline
\end{tabular}

is the optimizing prescription of TCM syndrome element "turbid phlegm" for patients with UA.

For commonly used names of Chinese herbal medicine, see Table 4.

\section{Discussion}

With the development of TCM, researches in optimizing prescription of Chinese herbal medicine are concerned by more and more scholars. Clinical formulation optimization is the process of using certain methods to improve clinical medicine prescriptions and make them applied in clinical practice. The commonly used methods at present are mainly two approaches as generalizing empirical formulation after optimization and applying data mining model to promote the optimized formulation [10].

The target of empirical formulation optimization is to study the experienced prescriptions promoted by some expert or clinician by modern medical research methods such as randomized control trail (RCT) in order to prove the reliability and practicality, providing evidence for related field of formulation optimization. This method is more stringent and convincing in formulation optimization design, and the optimizing prescriptions for the study are derived from the established treatment ones. However, cases collecting, observing, and other series of clinical research process need a large amount of human and material resources, which increases the cost of study; these many prescriptions with very good clinical applications are difficult to discover and research. In addition, for the more rigorous research process, the applicable conditions of the results received strict limits. In clinical practice, the efficacy in patients is affected by a variety of complex factors, which should be the results of a variety of drugs or interventions, making the extrapolation of experience prescriptions subject to certain restrictions.
Applying data mining model to optimize the prescriptions has got manifold attempts in clinical practice [11-14]; the most common method is a dynamic programming strategy. Its purpose is to seek the best solution in many prescriptions applying optimization techniques. Its data are from the clinical data of real world, and the data collection is carried out in parallel with the clinical course. As long as establishing the regulation of data entry, the entire data acquisition does not require large-scale acquisition process, and the time is mainly spent in data computing, which greatly saves the cost of the study to facilitate the continuous optimization of the prescriptions. Furthermore, this dynamic process is a combination of man and machine model; the strict mathematical operation is carried out at the same time when doing empirical evaluation. The actual process can be combined with expert consensus and evaluation to select the best treatment prescriptions given by computer for the most appropriate patient. This process can also be used for the discovery of clinical prescriptions or provide a scientific and rational arithmetic verification process for the summary of experience prescriptions, which may be very meaningful in the future.

POMDP model [15] is a dynamic decision model based on Markov process promoted by the Russian mathematician Markov after some improvements. Hauskrecht and Fraser [16] used POMDP in treatment prescription of ischemic heart disease and did a decision successfully in many cases. Maillart et al. [17] made analysis in the frequency of X-rays and treatment options for breast cancer patients from the costbenefit viewpoint with this model. Zhang et al. [18] established POMDP model for prostate biopsy decisions. These explorations all provide practical basis for solving problems of sequence decision with POMDP model in the medical field. This study selected this research strategy to optimize prescriptions of Chinese herbal medicine for patients with $\mathrm{UA}$, which is an exploration on the methodology. 
TABLE 4: Commonly used names of Chinese herbal medicine.

\begin{tabular}{|c|c|c|}
\hline Chinese name & English name & Latin name \\
\hline Dangshen & Tangshen & Radix Codonopsis \\
\hline Huangqi & Milkvetch Root & Radix Astragali \\
\hline Fuling & Indian Bread & Poria \\
\hline Baizhu & Largehead Atractylodes Rhizome & Rhizoma Atractylodis Macrocephalae \\
\hline Danshen & Danshen Root & Radix Salviae Miltiorrhiae \\
\hline Danggui & Chinese Angelica & Radix Angelicae Sinensis \\
\hline Honghua & Safflower & Flos Carthami \\
\hline Chishao & Red Peony Root & Radix Paeoniae Rubra \\
\hline Chuanxiong & Szechwan Lovage Rhizome & Rhizoma Chuanxiong \\
\hline Zhiqiao & Orange Fruit & Fructus Aurantii \\
\hline Gualou & Snakegourd Fruit & Fructrs Trichosanthis \\
\hline Xiebai & Longstamen Onion Bulb & Bulbus Allii Macrostemi \\
\hline Banxia & Pinellia Tuber & Rhizoma Pinelliae \\
\hline Chenpi & Dried Tangerine peel & Pericarpium Citri Reticulatae \\
\hline Jiegeng & Platycodon Root & Radix Platycodi \\
\hline Taizishen & Heterophylly Falsestarwort Root & Radix Pseudostellariae \\
\hline Gancao & Radix Glycyrrhizae & Radix Glycytthizae \\
\hline Dazao & Chinese Date & Fructrs Jujubae \\
\hline Huanglian & Golden Thread & Rhizoma Coptidis \\
\hline Zhuru & Bamboo Shavings & Caulis Bambusae in Taeniam \\
\hline Huoxiang & Wrinkled Giant Hyssop & Agastache rugosa \\
\hline Peilan & Herba Eupatorii & Eupatorium fortunei \\
\hline Yanhusuo & Yanhusuo & Rhizoma \\
\hline Taoren & Peach Seed & Semen Persicae \\
\hline Yujin & Turmeric Root Tuber & Radix Curcumae \\
\hline Baishao & White peony Alba & Raidix Paeoniae Alba \\
\hline Chaihu & Radix bupleuri & Bupleurum chinense \\
\hline Shengdi & Rehmanniae radix & Rehmannia glutinosa Libosch \\
\hline
\end{tabular}

Concerning POMDP modeling requirements, this study was modeled following the dynamic clinical decision-making process to make the degree of remission in the symptoms for patients with UA and long-term end-point events as efficacy evaluation indicators for the choice of the optimal prescriptions; at the same time, it evaluated the original core prescription and extracted optimal treatment prescriptions as optimization recommendations of patients with UA in a certain TCM syndrome element for clinical reference.

Based on existing data, applying POMDP to compare the prescriptions of patients with same TCM syndrome element and no long-term end-point event, we found that optimizing prescription recommendation for " $q i$ deficiency" patients is "Milkvetch Root $+\mathrm{Si}$ junzi decoction without Radix Glycyrrhizae," prescription of "blood stasis" recommended "Danshen Root + Tao Hong Siwu decoction plus Orange Fruit without rehmanniae radix," prescription of "turbid phlegm" recommended "Gualou xiebai banxia decoction plus Dried Tangerine peel, Largehead Atractylodes Rhizome, Platycodon Root". Those are recommendations from strict mathematical model stimulating dynamic TCM prescription process, which are in line with conventional clinical thinking and put forward proposals worthy of strict clinical research.

Besides that, the prescriptions derived from real clinical data are experiences and summaries of clinical practice with considerable clinical significance. The proposals are in conformity with the clinical normal circumstances and prove the reliability and operability of optimizing prescription method in efficacy evaluation on the other hand.

It should be noted that the rigorous mathematical comparison method using in this study to observe short-term and long-term efficacy of prescriptions in order to discover preliminary optimizing recommendations is limited to the number of patients, indicators, follow-up time, and so on that the extension value should apply modern medical research methods such as case-control trail to do retrospectively summarized comparison or large-scale, multicenter, and large sample RCT for further verification to increase the level of evidence-based medicine. However, in complex data of clinical practice, the efficacy of the patients is affected by many complicated factors; this formulation optimization idea still has great significance in efficacy comparison and screening treatment plan, and it also provides us an optimizing prescription method for clinical practice-based data worthy of further study.

\section{Acknowledgments}

The current work was partially supported by Beijing Committee of Science and Technology (no. D08050703020801), 
the TCM Public Welfare Scientific Research Project, State Administration of TCM of People's Republic of China (no. 201007001), the National Key Basic Research Prescription of China (no. 2006CB504803), the Twelve Five-year Plan of China (nos. 2013BAI02B01 and 2013BAI13B01), and Dean's Fund of Beijing Anzhen Hospital affiliated to Capital Medical University (2013Z05).

\section{References}

[1] Y. Z. Chen, Practical Internal Medicine, Beijing People's Health Publishing House, 11th edition, 2001.

[2] E. J. Sondik, The Optimal Control of Partially Observable Markov Processes, Department of Electrical Engineering, Stanford University, Stanford, Calif, USA, 1971.

[3] R. D. Smallwood and E. J. Sondik, "The optimal control of partially observable Markov processes over a finite horizon," Operations Research, vol. 21, no. 5, pp. 1071-1088, 1973.

[4] E. J. Sondik, "The optimal control of partially observable Markov processes over the infinite horizon: discounted costs," Operations Research, vol. 26, no. 2, pp. 282-304, 1978.

[5] China Cardiovascular Association, "Diagnosis and treatment guidelines of Unstable angina and non-ST-segment elevation myocardial infarction," Chinese Medical Journal, vol. 35, no. 4, pp. 295-304, 2007.

[6] China Association of Integrative Medicine Association for Cardiovascular Diseases, "TCM standards of coronary heart disease," Chinese Journal of Integrated Traditional and Western Medicine, vol. 11, no. 5, p. 257, 1991.

[7] Y. Y. Wang, Chinese Medical Science, Shanghai Science and Technology Press, Shanghai, China, 6th edition, 2001.

[8] T. T. Deng, Diagnostics of Chinese Medicine (Revised Edition), Shanghai Science and Technology Press, Shanghai, China, 2007.

[9] N. L. Yao, Syndromes Differential Diagnosis of Traditional Chinese Medicine, People's Health Publishing House, Beijing, China, 2nd edition, 2002.

[10] Y. M. Xie, Y. Y. Wang, and W. L. Wen, "Ideas and methods of clinical medical prescription optimization," World Science and Technology: Modernization of Traditional Chinese Medcine, vol. 10, no. 1, pp. 22-26, 2008.

[11] J. Poon, Z. Luo, and R. Zhang, "Feature representation in the biclustering of symptom-herb relationship in Chinese medicine," Chinese Journal of Integrative Medicine, vol. 17, no. 9, pp. 663-668, 2011.

[12] X. Z. Zhou, R. S. Zhang, J. Shah et al., "Patterns of herbal combination for the treatment of insomnia commonly employed by highly experienced Chinese medicine physicians," Chinese Journal of Integrative Medicine, vol. 17, no. 9, pp. 655-662, 2011.

[13] L. Zhang, D. L. Yu, Y. G. Wang, and Q. Zhang, "Selecting an appropriate interestingness measure to evaluate the correlation between Chinese medicine syndrome elements and symptoms," Chinese Journal of Integrative Medicine, vol. 18, no. 2, pp. 93-99, 2012.

[14] T. Chen, X. Z. Zhou, R. S. Zhang, and L. W. Zhang, "Discovery of regularities in the use of herbs in Chinese medicine prescriptions," Chinese Journal of Integrative Medicine, vol. 18, no. 2, pp. 88-92, 2012.

[15] X. M. An and L. Lin, "Markov model research progress in the vital statistics," Chinese Journal of Health Statistics, vol. 24, no. 4, pp. 436-439, 2007.
[16] M. Hauskrecht and H. Fraser, "Planning treatment of ischemic heart disease with partially observable Markov decision processes," Artificial Intelligence in Medicine, vol. 18, no. 3, pp. 221244, 2000.

[17] L. M. Maillart, J. S. Ivy, S. Ransom, and K. Diehl, "Assessing dynamic breast cancer screening policies," Operations Research, vol. 56, no. 6, pp. 1411-1427, 2008.

[18] J. Zhang, B. T. Denton, H. Balasubramanian, N. D. Shah, and B. A. Inman, "Optimization of PSA screening policies: a comparison of the patient and societal perspectives," Medical Decision Making, vol. 32, no. 2, pp. 337-349, 2012. 


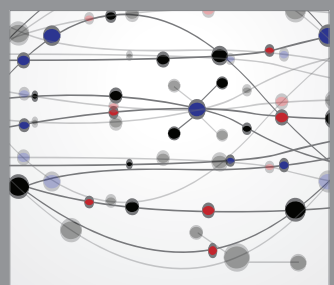

The Scientific World Journal
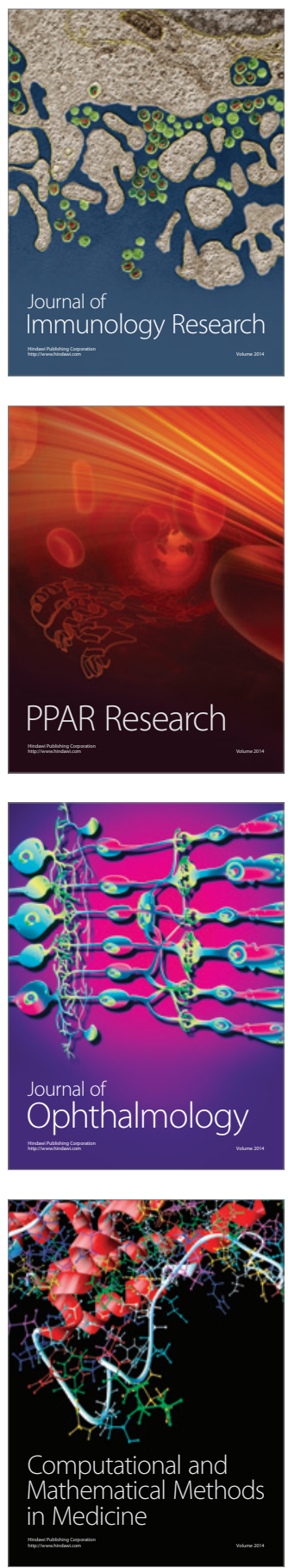

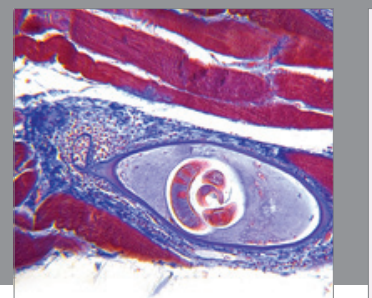

Gastroenterology

Research and Practice
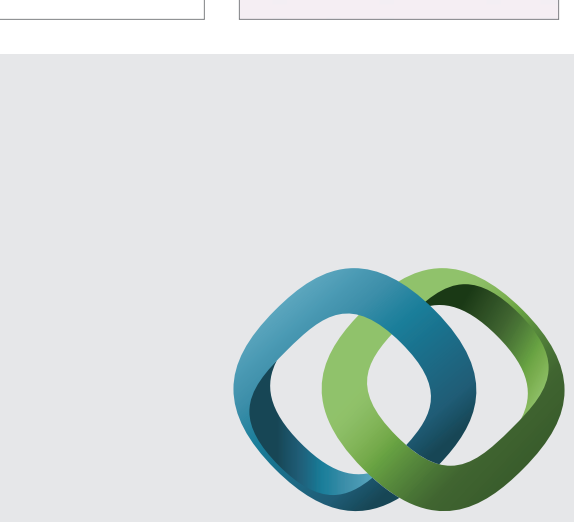

\section{Hindawi}

Submit your manuscripts at

http://www.hindawi.com
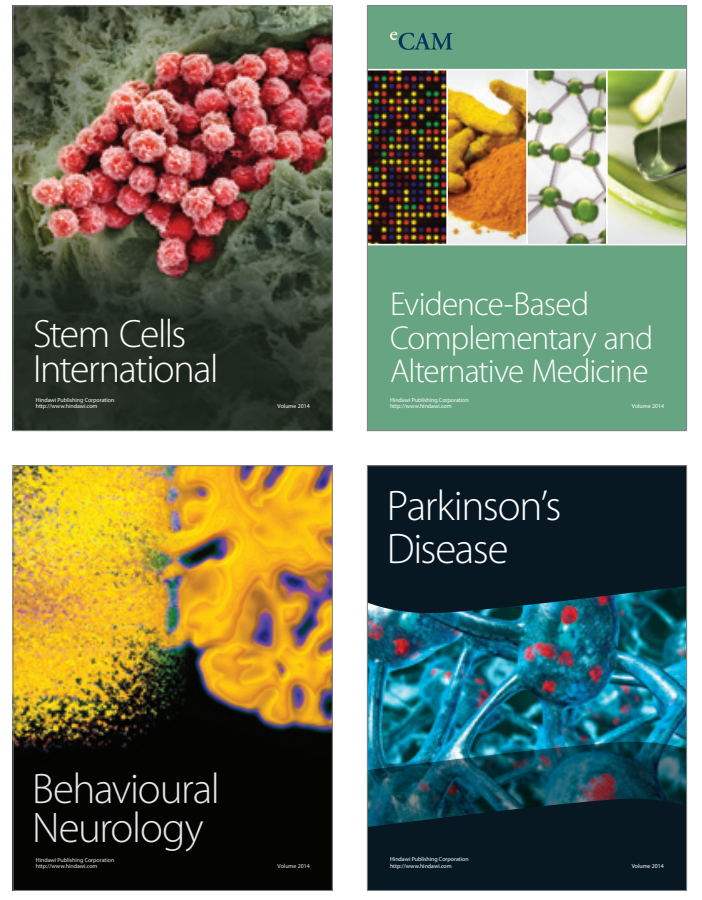
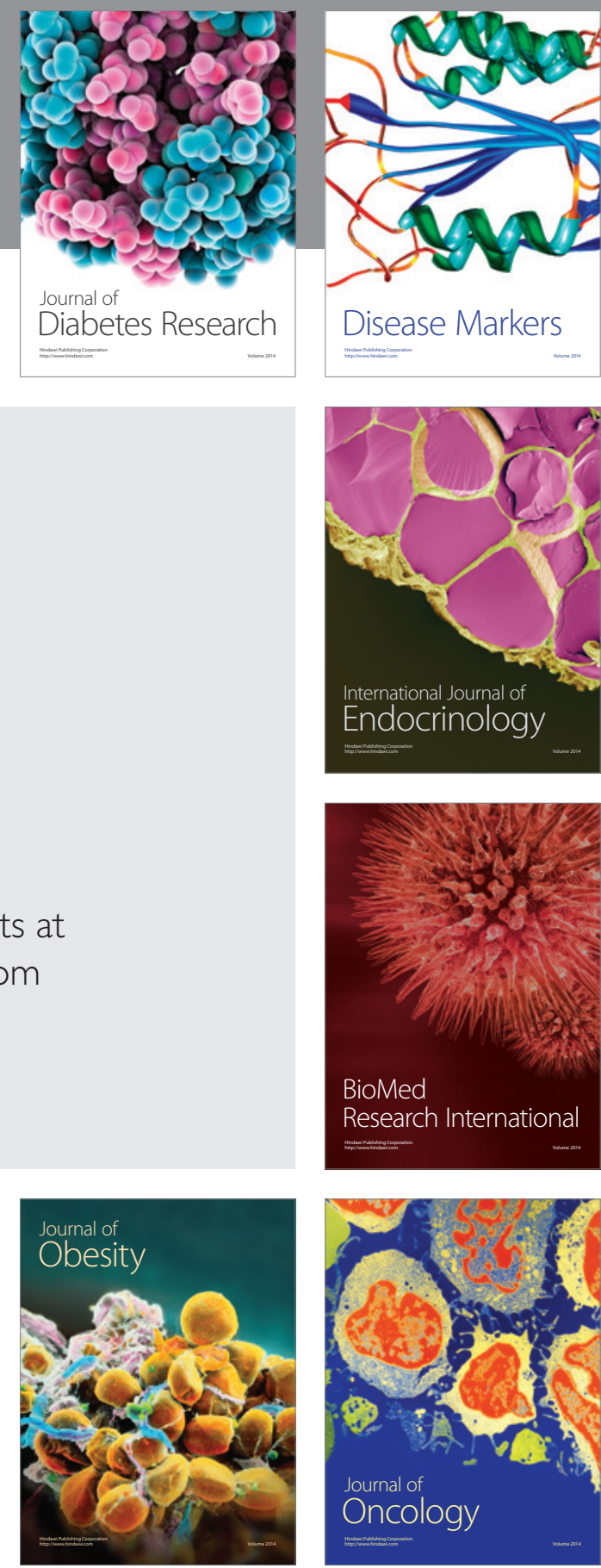

Disease Markers
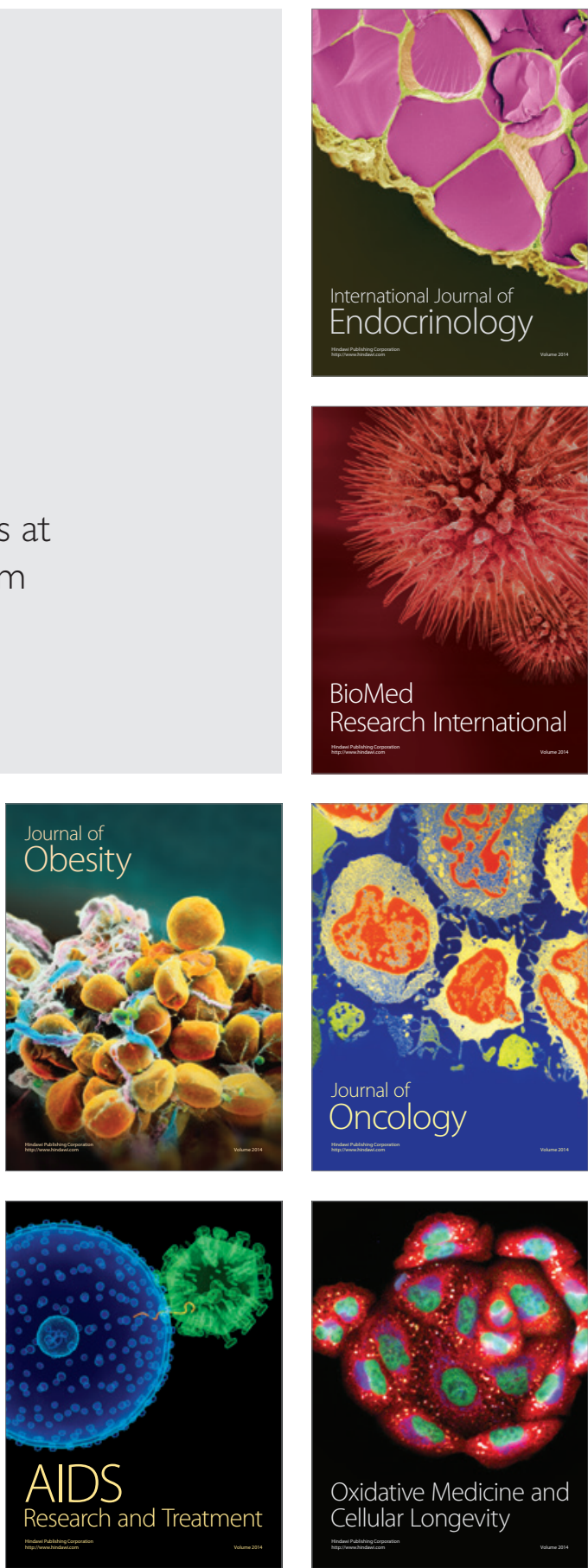